\title{
THE LINEARITY OF THE COSMIC EXPANSION FIELD AND THE VALUE OF THE HUBBLE CONSTANT
}

\author{
G. A. TAMMANN \\ Astronomisches Institut der Universität Basel, \\ Venusstr. 7, CH-4102 Binningen, Switzerland
}

\begin{abstract}
A linearity test shows $H_{0}$ to decrease by $7 \%$ out to $18000 \mathrm{~km} \mathrm{~s}^{-1}$. The value at $10000 \mathrm{~km} \mathrm{~s}^{-1}$ is a good approximation to the mean value of $H_{0}$ over very large scales. The construction of the extragalactic distance scale is discussed. Field galaxies, cluster distances relative to Virgo, and blue supernovae of type Ia yield $H_{0}$ (cosmic) with increasing weight; they give consistently $H_{0}=57 \pm 7$ (external error). This value is supported by purely physical distance determinations (SZ effect, gravitational lenses, MWB fluctuations). Arguments for $H_{0}>70$ are discussed and shown to be flawed.
\end{abstract}

\section{Introduction}

The calibration of the cosmic expansion rate $H_{0}$ consists of two steps. The first step is an investigation of the cosmic expansion field. How linear is the expansion? How large are systematic deviations from linearity in function of distance? What is the scatter of individual objects due to peculiar motions about the mean expansion? Only after these questions are solved can the second step be tackled, i. e. the calibration of the expansion rate in absolute terms.

The procedural difference between the two steps is that only redshifts and relative distances are needed for an investigation of the characteristics of the expansion field, while the calibration of the present large-scale expansion rate $H_{0}$ requires in addition the true distance of at least one object which demonstratably partakes of the mean expansion.

Much confusion about the expansion rate has arisen from equating the velocity-distance ratio of a subjectively chosen object with $H_{0}$. The determination of $H_{0}$ from the Virgo, Fornax, or Coma clusters, for instance, 
is meaningful only if it is demonstrated that they reflect at their moderate distances the mean cosmic expansion. The long-standing problem of correcting the observed mean velocity of the Virgo cluster into the frame of the cosmic expansion field has become a classic (cf. Section 3.2). The Fornax cluster with a velocity of $v \approx 1200 \mathrm{~km} \mathrm{~s}^{-1}$ cannot be used for the determination of $H_{0}$, even if a useful distance was known for it, because its unknown peculiar velocity may well be as high as $20 \%$ of its observed velocity. And the Coma cluster at $v \approx 7000 \mathrm{~km} \mathrm{~s}^{-1}$, which is sometimes used for the determination of $H_{0}$, may still have a peculiar velocity component of $10 \%$, as the peculiar velocity of $630 \mathrm{~km} \mathrm{~s}^{-1}$ with respect to the MWB of one other supercluster, i. e. the Local Supercluster, would suggest.

The present paper outlines this two-step procedure. In Section 2 the available data are used to map the expansion rate in function of distance well beyond $30000 \mathrm{~km} \mathrm{~s}^{-1}$, i.e. out to distances where the truly cosmic character of $H_{0}$ cannot be questioned. Section 3 gives a summary of the various methods of determining distances of field galaxies, of the Virgo cluster, and - most decisively for $H_{0}$ - of distant blue SNe Ia. Methods leading seemingly to $H_{0}>70$ are critically discussed in Section 4. A brief outlook is given in Section 5 .

\section{The Cosmic Expansion Field}

Hubble (1929) in his discovery paper plotted recession velocities versus linear distances to infer the expansion of the Universe. All subsequent papers on the subject have used instead a plot of $\log c z$ versus $\log$ (distance), the advantage being that only distance ratios are needed. The resulting diagrams have become known as Hubble diagrams. As a measure of $\log$ (distance) the apparent magnitude of standard candles, the apparent diameters of standard rods, or relative distance moduli $\Delta(m-M)$ can be used. Using the Hubble diagrams of different objects the overall linearity of the cosmic expansion field has been proven without doubt.

In the case of linear expansion the regression line of the Hubble diagram has slope 0.2 if apparent magnitudes of standard candles or relative distance moduli are used. Their Hubble diagrams allow therefore three additional tests:

(1) If a specific data set yields a slope different from 0.2 it is an unfailing indication that the distance scale is incorrect. (Uncorrected selection effects, i. e. Malmquist bias, always yield too steep slopes and a spurious increase of $H_{0}$ with distance).

(2) The scatter about the regression line is due to a combination of the effects of peculiar motions and errors of the relative distances. If the latter are under control one can determine the mean relative size $\Delta v / v_{\mathrm{c}}$ of the 
peculiar motions, where $v_{\mathrm{c}}$ is the cosmic velocity required by the mean Hubble line.

(3) If the relative peculiar motions $\Delta v / v_{c}$ are plotted against the distance $r$ (or in sufficient approximation against the recession velocity $v$ ) one can test for local deviations from perfect Hubble flow. If $H_{i}=\left(v_{\mathrm{c}}+\Delta v\right) / r$ is the perturbed value of the Hubble ratio of the $i$-th object at distance $r$, and $H_{0}=<H_{i}>=v_{\mathrm{c}} / r$ the true Hubble constant, than $\Delta H / H_{0}=$ $\left(H_{i}-H_{0}\right) / H_{0}=\Delta v / v_{\mathrm{c}}$. This test of relative variation of $H_{0}$ is performed in the following.

Three independent data sets are used for the test, viz. the Hubble diagram of first-ranked cluster galaxies (Sandage et al., 1976), the Hubble diagram of blue SNe Ia (Saha et al., 1997; slightly updated by Parodi and Tammann, 1998) and the Hubble diagram with 31 relative cluster distances (Federspiel et al., 1998). In each Hubble diagram the residuals $\Delta v / v_{\mathrm{c}}$ are read and combined within $5000 \mathrm{~km} \mathrm{~s}^{-1}$ bins. Sliding means in $2500 \mathrm{~km} \mathrm{~s}^{-1}$ steps are plotted in Fig. 1 against redshift.

Inspection of Fig. 1 strongly suggests that the Hubble constant decreases from $1000 \mathrm{~km} \mathrm{~s}^{-1}$ to about $18000 \mathrm{~km} \mathrm{~s}^{-1}$ by $\sim 7 \%$. This trend is independently supported by the first-ranked cluster galaxies of Lauer and Postman (1994), which have not been used here. Beyond $18000 \mathrm{~km} \mathrm{~s}^{-1}$ the scatter becomes large, leaving the possibility of local $\pm 10 \%$ variations of $H_{0}$, but the distant overall mean of $H_{0}$ lies close to the value found at $10000 \mathrm{~km} \mathrm{~s}^{-1}$.

The consequences for the calibration of $H_{0}$ are clear. Full-sky samples with $v>1000 \mathrm{~km} \mathrm{~s}^{-1}$ yield the cosmic value of $H_{0}$ to within $\pm 5 \%$. The best mean cosmic value is found near $10000 \mathrm{~km} \mathrm{~s}^{-1}$ or from sufficiently large samples beyond $20000 \mathrm{~km} \mathrm{~s}^{-1}$.

\section{The Calibration of $H_{0}$}

The interlaced construction of the distance scale out to $30000 \mathrm{~km} \mathrm{~s}^{-1}$ and beyond is schematically shown in Fig. 2. Brief comments on the individual steps, labelled (1) to (9), proceed as follows.

(1) The distance of LMC is the first fundamental step outward. The best values come from the purely geometrical distance of the ring of SN 1987A (Panagia et al., 1996), from Cepheids in various passbands, and RR Lyr stars. The zeropoint of the Cepheid P-L relation is based on Cepheids in Galactic clusters (Sandage and Tammann, 1971; Feast, 1995), on stellar radii (Di Benedetto, 1997), on the Baade-Becker-Wesselink method (Laney and Stobie, 1992) and on Hipparcos trigonometric parallaxes (Madore and Freedman, 1998; Sandage and Tammann, 1998). The zeropoint of the RR Lyr star P-L relation in function of metallicity comes from physical considerations (Sandage, 1993a), the Baade-Becker-Wesselink method (Sandage 

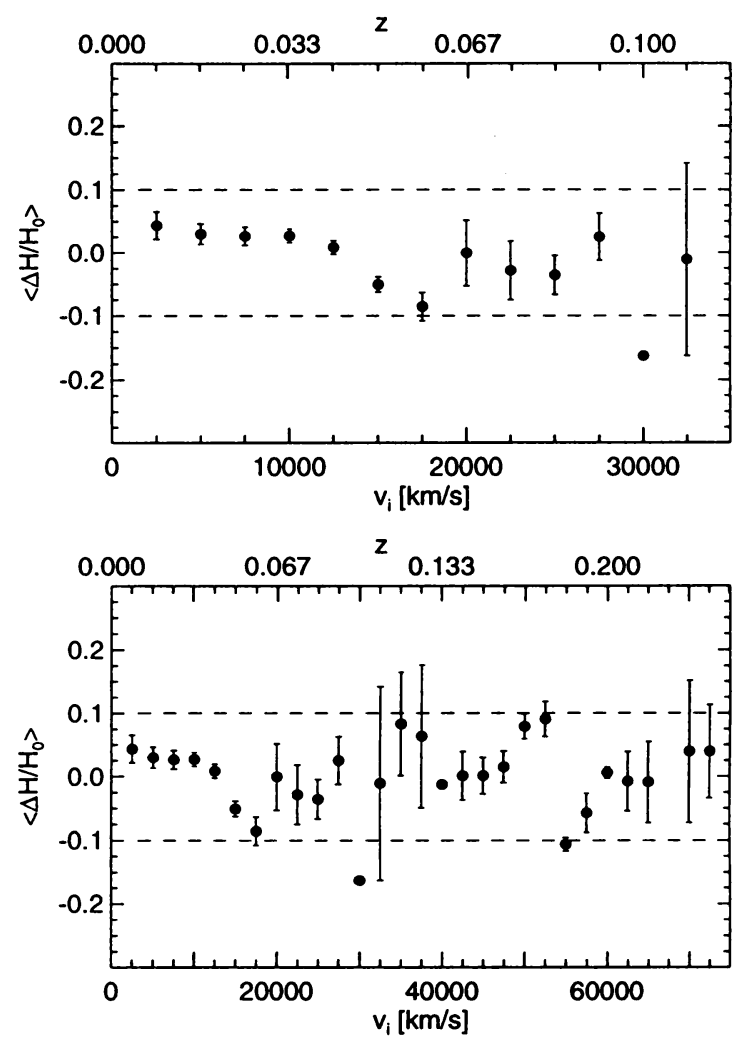

Figure 1. The variation of $H_{0}$ with redshift derived from relative distances. a) Out to $35000 \mathrm{~km} \mathrm{~s}^{-1}$ using relative cluster distances, $\mathrm{SNe} \mathrm{Ia}$, and first-ranked cluster galaxies. b) Out to $72500 \mathrm{~km} \mathrm{~s}^{-1}$; beyond $35000 \mathrm{~km} \mathrm{~s}^{-1}$ the data depend only on first-ranked cluster galaxies.

and Cacciari, 1990), and from globular clusters fitted to the Hipparcoscalibrated main sequence of subdwarfs (Reid, 1998; Gratton et al., 1997; Pont et al., 1998). Individual LMC moduli are compiled in Federspiel et al. (1998) and give $(m-M)=18.54 \pm 0.03$. This value is also in good agreement with the position of the red-giant tip (Lee et al., 1993; Tammann, 1996). Conservatively $(m-M)=18.50$ has been adopted in the following. This value is secure to within $\pm 5 \%$ and is uncontroversial.

(2) The distance of LMC combined with the good photometry of many of its Cepheids yields the calibrated P-L relation at different wavelengths (Sandage and Tammann, 1971). For the $H S T$ observations in $I$ and $V$ the P-L relations of Madore and Freedman (1991) are generally used; this has the advantage that any divergence in the derived value of $H_{0}$ cannot be blamed on the use of different P-L relation. 


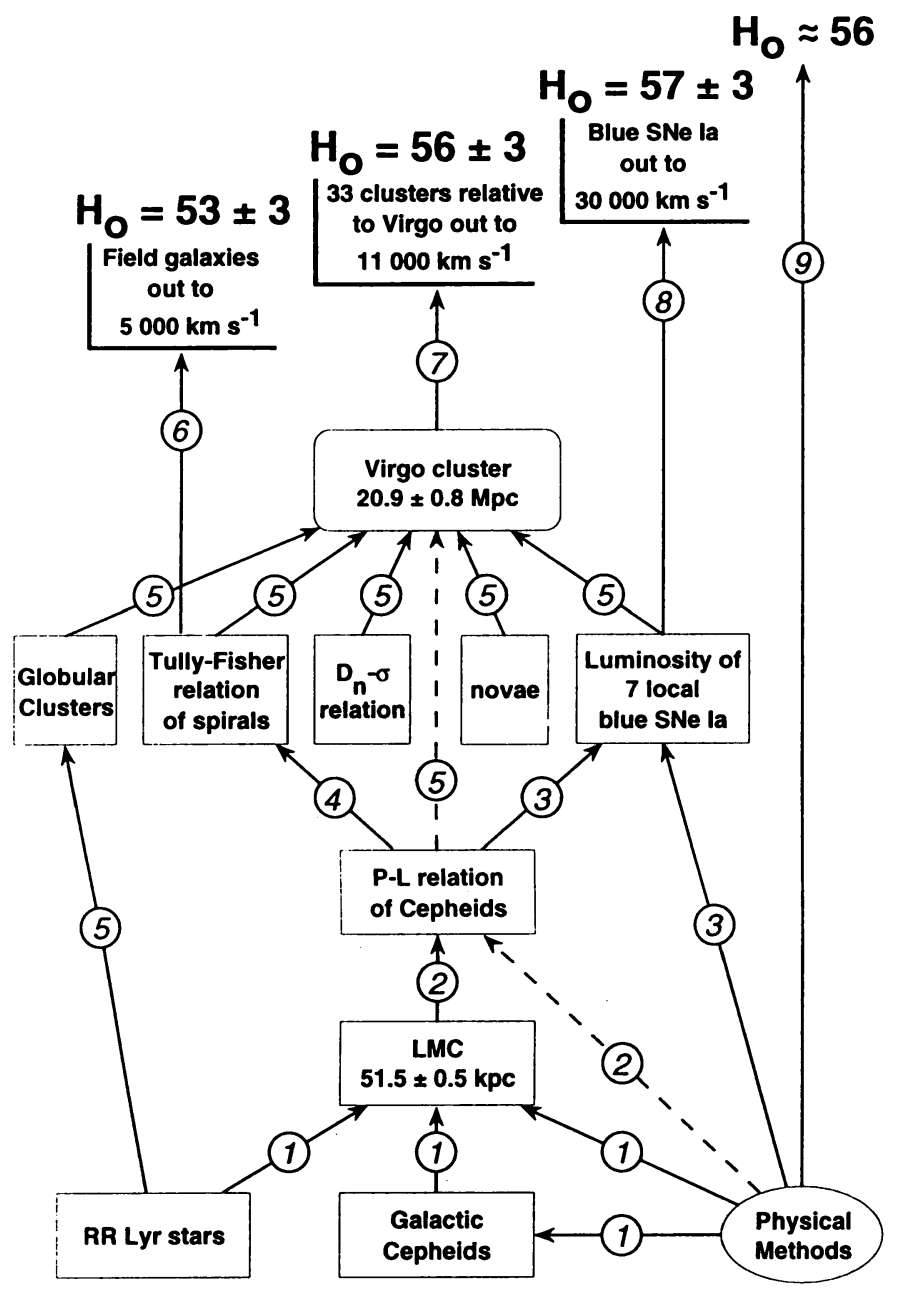

Figure 2. Schematic presentation of the interlaced extragalactic distance scale

Typical errors of individual Cepheid distances derived from $H S T$ data are $\pm 10 \%$ due to the width of the instability strip and restricted sample size and due to absorption. For the luminosity calibration of SNe Ia (cf. (8) the errors are smaller because only apparent distance moduli are used. - Attempts to improve Cepheid distances with the help of a periodluminosity-color (PLC) relation (e.g. Kochanek, 1997) are doomed because stellar evolution models combined with a pulsation code show that the basic assumptions going into a PLC relation are not met (Saio and Gautschy, 1998). The same models show that the much-discussed metallicity has minimal effect on the $\mathrm{P}-\mathrm{L}_{\text {bol }}$ relation; remaining metallicity effects enter only 
through the bolometric correction.

(3) The Cepheid distances from $H S T$, most relevant for $H_{0}$, concern the six nearby galaxies which have produced seven well studied blue (i. e. "Branch - normal") SNe Ia (cf. (8)). Their mean luminosity is (Saha et al., 1997; Saha, 1998) $M_{\mathrm{B}}(\max )=-19.54, M_{\mathrm{V}}(\max )=-19.50$ with an impressively small scatter of $0 \mathrm{~m}_{16} 16$ which - in addition to the arguments presented in (8) - demonstrates that blue SNe Ia are highly useful standard candles.

The luminosity of SNe Ia can also be determined from physical explosion models. The state-of-the-art light curve fitting method by Höflich and Khokhlov (1996) give $M_{\mathrm{B}}(\max )=-19.45$ for the $16 \mathrm{SNe}$ Ia to which sufficiently blue models can be fitted. In a review of all physical determinations of $M(\max )$ Branch $(1998)$ concludes $M_{\mathrm{B}}(\max ) \approx M_{\mathrm{V}}(\max ) \approx-19.4-19.5$. The close agreement of the astronomical and physical calibrations of blue SNe Ia is most encouraging.

(4) The calibration of the $21 \mathrm{~cm}$ line width-luminosity (Tully-Fisher, TF) relation in the B-band can now be based on 18 Cepheid distances most of which are due to $H S T$. They determine the zeropoint of the relation with a statistical error of only $0{ }^{\mathrm{m}} 05$, but they are still not enough for the determination of the slope. The latter must be taken from a complete sample of 49 inclined spirals of the Virgo cluster (Federspiel et al., 1998).

In principle redder wavelengths than the $\mathrm{B}$ band offer the advantage of smaller inclination-dependent absorption corrections. However, this advantage is entirely offset by the steeper slope of the $\mathrm{TF}$ relation at longer wavelengths (Schröder, 1996). Moreover the restricted number of available magnitudes at longer wavelengths and their possible inhomogeneity, particularly in the $I$ and $H$ bands, preclude the all-important construction of complete samples. Therefore absolute TF distances of the Virgo cluster and of field galaxies are problematic if derived from magnitudes other than $B$. (5) The distance of the Virgo cluster can be derived, as shown in Fig. 2, in six different ways:

a) Cepheid distances give so far only a rather poorly determined cluster distance. The reason is the important depth effect of the cluster. Four of the five galaxies with distances from Cepheids have been selected because they are well or even extremely well (NGC 4571) resolved and expected to lie on the near side of the cluster. Their mean distance is $16.1 \pm 0.4 \mathrm{Mpc}$ (Freedman et al., 1998). One galaxy, NGC 4639, has been selected irrespective of resolution and its distance is $25.1 \pm 2.5 \mathrm{Mpc}$ (Saha et al., 1997). The position of the four resolved galaxies on the near side and of the poorly resolved NGC 4639 on the far side of the cluster is independently confirmed by their relative TF distances (Federspiel et al., 1998). One can therefore infer only that the center of the cluster lies roughly at $(m-M)=31.5 \pm 0.5$ $(20 \mathrm{Mpc})$. A preliminary way out is to use the mean distance of the Leo 
TABLE 1. The Virgo cluster modulus from various methods

\begin{tabular}{lll}
\hline Method & $(m-M)_{\text {Virgo }}$ & Hubble type \\
\hline Cepheids & $31.52 \pm 0.21$ & S \\
SNe Ia & $31.39 \pm 0.17$ & E, S \\
Tully-Fisher & $31.58 \pm 0.24$ & S \\
Globular Clusters & $31.67 \pm 0.15$ & E \\
Dn $-\sigma$ & $31.85 \pm 0.19$ & So, S \\
Novae & $31.46 \pm 0.40$ & E \\
\hline Mean: & $31.60 \pm 0.08$ & $(\Rightarrow 20.9 \pm 0.8 \mathrm{Mpc})$ \\
\hline
\end{tabular}

group of $(m-M)=30.27 \pm 0.12$, based now on three galaxies with Cepheid distances, and to step up this value by the modulus difference of $\Delta(m-M)=1.25 \pm 0.13$ (Tammann and Federspiel, 1997) between the Leo Group and the Virgo cluster, giving $(m-M)_{\text {Virgo }}=31.52 \pm 0.21$.

b) Eight SNe Ia with known maximum magnitudes are available. Three of these have occurred in E/S0 galaxies; they are known to be underluminous by $0{ }^{\mathrm{m}} 18$. If the latter are adjusted to $\mathrm{SNe} \mathrm{Ia}$ in spirals to conform with the calibrating SNe Ia, the mean apparent magnitude of the eight SNe Ia becomes $\left\langle m_{B}(\max )\right\rangle=11.91 \pm 0.16$ and $\left\langle m_{V}(\max )\right\rangle=11.84 \pm 0.17$. With the calibration in (3) the mean cluster modulus becomes then $31.39 \pm 0.17$. c) The TF relation in $B$ magnitudes of a complete sample of 49 cluster galaxies, combined with the TF calibration in (4), gives $(m-M)_{\mathrm{Virgo}_{\mathrm{r}}}=$ $31.58 \pm 0.24$ (Federspiel et al., 1998). The relatively large error allows for a number of systematic effects like sample selection, propagation of observational errors, and inclination and color differences between calibrators and cluster galaxies.

$d-f)$ Other useful determinations of the Virgo cluster distance come from the luminosity function of globular clusters, the $\mathrm{D}_{\mathrm{n}}-\sigma$ relation of the bulges of S0 and spiral galaxies, and from novae. Because of the restricted space here the reader is referred to Sandage and Tammann (1997) and Tammann and Federspiel (1997). The results of the six methods a) -f) are compiled in Table 1.

The surface brightness fluctuation (SBF) method is sometimes advertised as a distance indicator. However, its reliability has not been sufficiently demonstrated yet for distances beyond $10 \mathrm{Mpc}$. The SBF distance of NGC 7331 (Tonry, 1997) is 0 555 smaller than its Cepheid distance (Hughes, 1997), and the proposed Virgo cluster modulus (Tonry, 1997) is smaller than that in Table 1 by the same amount. The applicability of the method will still depend on a large sample of Virgo cluster members to decide if 
the same relation applies for ellipticals and bulges of spiral galaxies. If not, the zeropoint calibration of the method will depend on an adopted Virgo cluster distance.

It has also been suggested that the luminosity function of the shells of planetary nebulae (PNe) in the light of the $\lambda 5004 \AA$ line had a magic cutoff luminosity which could be used as a universal distance indicator. However, it has been shown that the available data are at least equally consistent with a roughly exponential bright tail of the luminosity function such that the brightness of the brightest PNe depends on the sample size, i.e. on the luminosity of the galaxy under consideration (Bottinelli et al., 1991; Tammann, 1993). This conclusion has been buttressed by model calculations by Soffner et al. (1996). The recent proposal of a Virgo modulus of $(m-M)=30.79 \pm 0.16$ (Ciardullo et al., 1998), based on a simple cutoff assumption, is significantly smaller than the Cepheid distance of even the nearest, highly resolved Virgo spirals (cf. (5)a) and is therefore self-defeating. (6) The route to $H_{0}$ through field galaxies is the most difficult one. The crux is selection bias. Its origin is the fact that astronomers work with magnitude-limited catalogs of field galaxies, in which case the mean luminosity of the catalogued objects increases with distance. The disastrous consequences of selection (Malmquist) bias are illustrated, for instance, by a realistic model calculation by Hendry and Simmons (1990). They show that denying the selection bias can lead to $H_{0}=80$, while proper allowance for bias - depending on the luminosity scatter of the galaxies - yields values of $H_{0}=56$ or even $H_{0}=44$. Two fundamental facts emerge from this. Neglect of the selection bias always leads to too high values of $H_{0}$, and the severity of the error is a strong function of the luminosity scatter. In the case of cluster distances one can overcome the problem by working with complete samples within a given volume (cf. (7)). Blue SNe Ia offer the very important advantage that their luminosity scatter is so small that $H_{0}$ will not be significantly overestimated (cf. (8)).

Analytical corrections for Malmquist bias require knowledge of the true scatter, which can only be derived from very deep samples, and must neglect the clumpy distribution of galaxies. Practical ways to correct for bias are, e. g., by Bottinelli et al. (1986a; 1986b), Lynden-Bell et al. (1988), Sandage (1988), Federspiel et al. (1994), Sandage et al. (1995), Giovanelli (1997a), and Theureau et al. (1997). A tutorial on the subject is given by Sandage (1995).

Recent determinations of $H_{0}$ from bias-corrected field galaxies are compiled in Table 2. Field galaxies offer the advantage of full-sky coverage outside the zone of avoidance. But they are not only the most difficult route to $H_{0}$, but also the least satisfactory, having their main thrust as close as $1000-3000 \mathrm{~km} \mathrm{~s}^{-1}$, i. e. at a distance where $H_{0}$ (local) may still be 
TABLE 2. $H_{0}$ from bias corrected field galaxies

\begin{tabular}{lll}
\hline Method & \multicolumn{1}{c}{$H_{0}$} & Source \\
\hline Tully-Fisher & $<60$ & Sandage 1994 \\
M 101 look-alike diameters & $43 \pm 11$ & Sandage 1993b \\
M 31 look-alike diameters & $45 \pm 12$ & Sandage 1993c \\
Spirals with luminosity classes & $56 \pm 5$ & Sandage 1996a \\
M 101, M 31 look alike luminosities & $55 \pm 5$ & Sandage 1996b \\
Tully-Fisher & $55 \pm 5$ & Theureau et al. 1997 \\
Galaxy diameters & $50-55$ & Goodwin et al. 1997 \\
Tully-Fisher & $60 \pm 5$ & Federspiel 1998 \\
\hline mean & $53 \pm 3$ & \\
\hline
\end{tabular}

a few percent higher than $H_{0}$ (cosmic) (cf. Fig. 1).

(7) $H_{0}$ (cosmic) from clusters out to $11000 \mathrm{~km} \mathrm{~s}^{-1}$. Cluster distances relative to the Virgo cluster have been compiled from the literature (Jerjen and Tammann, 1993). The sample is increased by relative $I$-band TF distances of clusters (Giovanelli, 1997b). These relative distances are excellent as seen from their small scatter about the Hubble line of slope 0.2 in Fig. 3. The scatter is in fact so small that it imposes stringent limits on the radial component of the peculiar motion of cluster centers (Jerjen and Tammann, 1993).

The best fit to the data in Fig. 3 is

$$
\log c z=0.2\left[(m-M)_{\text {Cluster }}-(m-M)_{\text {Virgo }}\right]+(3.070 \pm 0.024)
$$

(Federspiel et al., 1998). From this follows directly

$$
\log H_{0}(\text { cosmic })=-0.2(m-M)_{\text {Virgo }}+(8.070 \pm 0.024) .
$$

Inserting $(m-M)_{\text {Virgo }}=31.60 \pm 0.08$ yields

$$
H_{0}(\operatorname{cosmic})=56 \pm 3 \quad \text { (internal error). }
$$

Note that no use of any velocity of the Virgo cluster has been made. The value of $H_{0}$ holds out to $\sim 10000 \mathrm{~km} \mathrm{~s}^{-1}$ where its value is very close to the large-scale value (Fig. 1).

(8) $H_{0}$ (cosmic) from blue SNe Ia. The Hubble diagram (in $V$ ) of 35 blue SNe Ia, mainly due to the heroic efforts of the Cerro Tololo group (Hamuy et al.,1996), is shown in Fig. 4. The small scatter $\sigma\left(m_{V}\right)$ of $0 \mathrm{~m}_{2} 4$ confirms the conclusion in (3) that blue SNe Ia are extremely useful standard candles. 


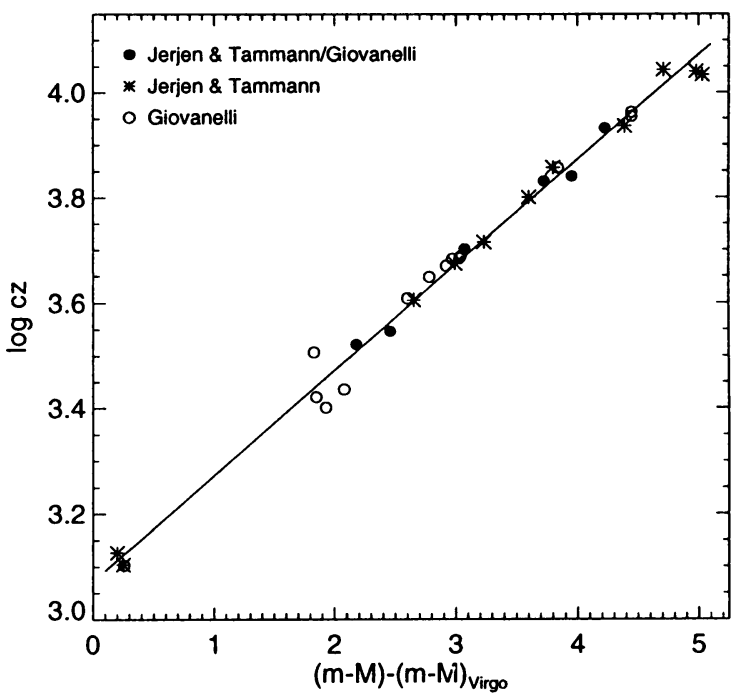

Figure 3. Hubble diagram of 31 clusters with known relative distances. Asterisks are data from Jerjen \& Tammann (1993). Open circles are from Giovanelli (1997a). Filled circles are the average of data from both sources.

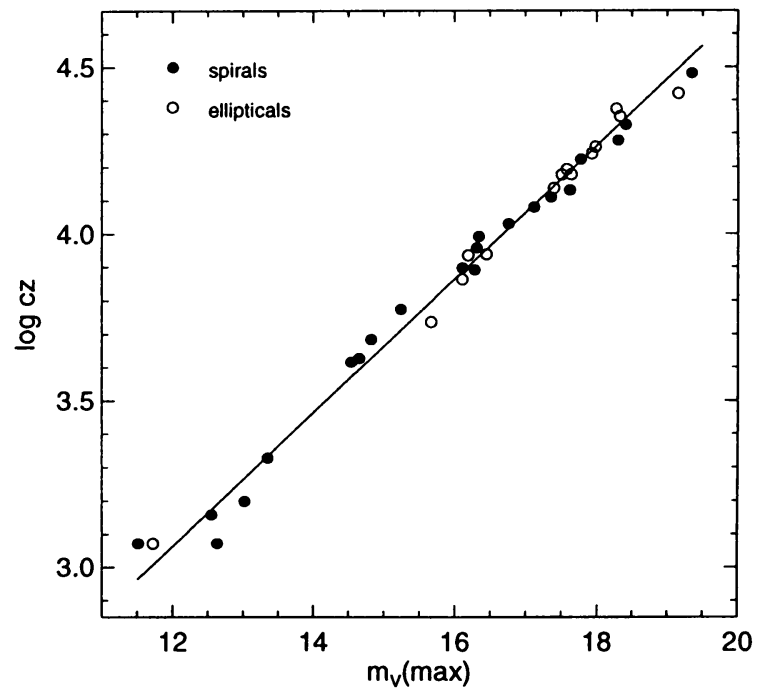

Figure 4. The Hubble diagram of 35 blue SNe Ia with photometry after 1985. The SNe Ia in elliptical galaxies are shifted by 0.18 . 
This is also supported by the absence of Malmquist bias, i.e. the Hubble line does not steepen with distance. A fit to the Hubble diagrams in $B$ and $V$ gives, after simple transformation,

$$
\begin{aligned}
& \log H_{0}=0.2 M_{B}+(5.651 \pm 0.011) \\
& \log H_{0}=0.2 M_{V}+(5.669 \pm 0.019) .
\end{aligned}
$$

These equations hold for SNe Ia in spirals. Those in $\mathrm{E} / \mathrm{S} 0$ galaxies are fainter on average by $0{ }^{\mathrm{m}} 18 \pm 0 \mathrm{~m} 05$ in $B$ and $V$ and have been shifted by this amount. Equations (4) and (5) can therefore directly be compared with the calibrators in spiral galaxies from (3). Thus one obtain a mean value from $B$ and $V$ data of

$$
H_{0}(\operatorname{cosmic})=57 \pm 3 .
$$

Red SNe Ia with $\left(B_{\max }-V_{\max }\right)>0.20$ have been excluded from the experiment because they are reddened or have peculiar spectra (Branch et al., 1993). Their unjustified inclusion leads to second-parameter corrections of the calibration in (3), but $H_{0}$ remains in all cases $<65$ (for details see Saha et al. 1997; cf. also 4.6). Within the claimed accuracy all secondparameter effects of blue SNe la have been taken care of by allowing for the underluminosity of $0 \mathrm{~m}_{1} 18$ of the $\mathrm{SNe}$ Ia in $\mathrm{E} / \mathrm{S} 0$ galaxies.

Of all present methods to derive $H_{0}$, the route through $\mathrm{SNe}$ Ia deserves the highest weight.

(9) $H_{0}$ (cosmic) from Physical Methods. One distinguishes between astronomical and physical distance determinations. The former depend always on some adopted distance of a celestial body, be it only the Astronomical Unit in the case of trigonometric parallaxes. Physical methods derive the distance solely from the observed physical or geometrical properties of a specific object.

In the foregoing reference has been made to physical luminosity and distance determinations of the SN 1987A remnant, Cepheids, RR Lyr stars, and SNe Ia. But in addition there are a number of physical distance determinations which lead to the value of $H_{0}$ over very large scales. They are still model-dependent, but as the number of objects increases and the models improve, their weight is steadily increasing.

For brevity the most recent physical determinations of $H_{0}$ are compiled in Table 3.

Following Rephaeli and Yankovitch (1997) all previous values of $H_{0}$ from the SZ effect should be lowered by $\sim 10$ units due to relativistic effects.

The overall impression from the values in Table 3 is that $H_{0}$ will settle around $H_{0} \approx 56$. 
TABLE 3. $H_{0}$ from Physical Methods

\begin{tabular}{clc}
\hline Method & $H_{0}$ & Source $^{a}$ \\
\hline Sunyaev-Zeldovich effect & & \\
for cluster A 2218 & $45 \pm 20$ & $(1)$ \\
for 6 other clusters & $60 \pm 15$ & $(2)$ \\
cluster A 2163 & $78(+54,-28)$ & $(3)$ \\
2 clusters & $42 \pm 10$ & $(4)$ \\
3 clusters & $54 \pm 14$ & $(5)$ \\
incl. relativ. effects & $44 \pm 7$ & $(6)$ \\
Gravitational lenses & & \\
QSO 0957+561 & $62 \pm 7$ & $(7)$ \\
B 0218 + 357 & $52-82$ & $(8)$ \\
PG 1115+080 & $60 \pm 17$ & $(9)$ \\
$"$ & $52 \pm 14$ & $(10)$ \\
$"$ & $62 \pm 20$ & $(11)$ \\
MWB fluctuation spectrum & $58 \pm 11$ & $(12)$ \\
& $47 \pm 6$ & $(13)$ \\
\hline
\end{tabular}

a Sources: (1) McHardy et al. 1990; Birkinshaw and Hughes 1994; Lasenby and Hancock 1995 (2) Rephaeli 1995; Herbig et al. 1995 (3) Holzapfel et al. 1997 (4) Lasenby and Jones 1997 (5) Myers et al. 1997 (6) Rephaeli and Yankovitch 1997 (7) Falco et al. 1997 (8) Nair 1996 (9) Keeton and Kochanek 1997 (10) Kundić et al. 1997 (11) Schechter et al. 1997 (12) Lineweaver 1998 (13) Webster et al. 1998

\section{4. $H_{0}=73$ ?}

Freedman et al. (1997) have proposed seven arguments why $H_{0}$ should be 73 (cf. also Freedman et al. 1998). These arguments are briefly discussed in the following.

4.1 $H_{0}=80 \pm 17$ from the Virgo cluster. This high value can only be suggested by combining a low cluster distance of $17.8 \mathrm{Mpc}$, relying heavily on Cepheids of near-side cluster members, and an outdated mean cluster velocity of $1404 \mathrm{~km} \mathrm{~s}^{-1}$. The best cluster velocity, corrected for all local effects, follows from equation (1) by setting $(m-M)_{\text {Cluster }}-(m-M)_{\text {Virgo }}=$ 0 , i. e. $1175 \pm 85 \mathrm{~km} \mathrm{~s}^{-1}$. This with $17.8 \mathrm{Mpc}$ gives $H_{0}=66$ and with a more realistic cluster distance from Table $1 H_{0}=56 \pm 4$.

4.2 $H_{0}=77 \pm 16$ from Coma via Virgo. The value depends, of course, on the adopted distance of the Virgo cluster. With a Virgo distance of $20.9 \pm 0.8$ (Table 1) one obtains $H_{0}=66 \pm 10$ with a large error due to the peculiar motion of the Coma cluster and to some extent also due to the 
error of the adopted relative distance Coma-Virgo.

$4.3 H_{0}=72 \pm 18$ from the Fornax cluster. The result comes from equating the single Cepheid distance of the spiral galaxy NGC 1365 of $18.4 \pm 1.0 \mathrm{Mpc}$ (Silbermann et al., 1998) with the mean cluster distance. There is no basis for this ad hoc assumption. A compilation of 30 distance determinations of the Fornax cluster over the last 20 years actually indicates that the $\mathrm{E} / \mathrm{S} 0$ members are $(1.17 \pm 0.05)$ times more distant than the spiral galaxies (Tammann and Federspiel, 1997). Taking this at face value one finds $21.5 \pm$ $1.5 \mathrm{Mpc}$ (cf. also 4.6) for the E/S0 galaxies and hence for the cluster center. This reduces the proposed value of $H_{0}=77 \pm 16$ to $H_{0}=66 \pm 15$ with a large uncertainty due to the unknown peculiar motion of the cluster (cf. Section 1).

4.4 $H_{0}=72 \pm 8$ from local data. The solution is dominated by the Virgo cluster (Sec. 4.1) and Fornax cluster (Sec. 4.3). The remaining three groups, NGC 2403, NGC 1023, and Leo, lie within $12 \mathrm{Mpc}$ and are irrelevant for the cosmic value of $H_{0}$.

$4.5 H_{0}=72 \pm 8$ from the Jerjen-Tammann clusters. The cluster sample has since been increased by the relative cluster distances of Giovanelli (1997; cf. Fig. 3 and equations (1) and (2)). With an (untenable) Virgo cluster modulus of $(m-M)=31.25 \pm 0.20$ from Sec. 4.1, equation (2) yields $H_{0}=66 \pm 7$ (not 72 !), but even this is too high because the inserted Virgo modulus is too small.

4.6 $H_{0}=67 \pm 8$ from SNe Ia. The authors follow in principle route (3) + (8), but they reject two calibrating SNe Ia on the ground of their photographic photometry (much of observational astronomy had to be discarded on that ground), and they speculate that SN 1980 and SN 1992A are at the same distance as NGC 1365. The two SNe Ia have occurred in the Fornax cluster E/S0 galaxies NGC 1316 and NGC 1380, respectively. As argued under 4.3, there is independent evidence that the $\mathrm{E} / \mathrm{S} 0$ galaxies in this cluster are more distant than the spiral NGC 1365. But for the sake of the argument the speculation of a common distance is taken up here. Since blue SNe Ia in early-type galaxies are fainter by 0.18 in $B$ and $V$ than their counterparts in spirals (cf. (8)), the mean absolute magnitude of SNe 1980 and 1992 becomes $M_{B}=-18.95$ and $M_{V}=-19.00$ if they lied at the distance of NGC 1365 and if they had occurred in spirals. This averaged in with the four remaining calibrators in (3) gives $\left\langle M_{B}(\max )\right\rangle=-19.31 \pm 0.12$ and $\left\langle M_{V}(\max )\right\rangle=$ $-19.33 \pm 0.11$, and inserted into equations (4) and (5), which hold for blue SNe Ia in spirals, yields a mean value of $H_{0}=62 \pm 6$. But even this rather low value is still internally inconsistent because the luminosity distribution of the six calibrators used becomes highly non-Gaussian, defying the basic conclusion of standard candles. It violates also the models of Höflich and Khokhlov (1996) for blue SNe Ia. It is much more plausible that SNe 1980N 
and 1992A have the same standard luminosity as found in (3), adjusted for early-type parent galaxies. In that case they require for the Fornax E/S0 galaxies $(m-M)=31.85(23.4 \mathrm{Mpc})$ in agreement with the independent value under 4.3 .

4.7 $H_{0}=73 \pm 7$ from the TF method. The authors base their claim on $I$ and $H$-band TF cluster distances of Mould et al. (1997) and Giovanelli et al. (1997). These authors consider highly incomplete and hence necessarily biased cluster samples, which may be useful for relative cluster distances (cf. also Kraan-Korteweg et al. 1988). In fact the relative distances of Giovanelli et al., who include a correction for differential bias, are found to be excellent (cf. Fig. 3). However, it is inadmissible to tie these biased subpopulations directly to the distance-limited sample of calibrators with known Cepheids. This necessarily leads to an underestimate of the cluster distances (Teerikorpi, 1987). It has been demonstrated, for instance, that the 25 sufficiently inclined Virgo galaxies with known $H$-magnitudes yield a cluster modulus $\sim 0{ }^{\mathrm{m}} 6$ smaller than the true value derived from a complete Virgo sample (Kraan-Korteweg et al. 1988; their Fig. 4). This discrepancy, which corresponds to a distance factor of $\sim 1.3$, perpetuates then through all cluster distances and immediately gives $H_{0} \approx 55$. The intermediate step through the Virgo cluster, i.e. from local calibrators to Virgo and from Virgo to more distant clusters, is necessary because the Virgo cluster is so far the only cluster for which a large and complete sample of spirals is available as well as extensive photometry and $21 \mathrm{~cm}$ data.

$4.8 H_{0}=73 \pm 7$ from physical models of SNe II. The authors cite the work of Schmidt et al. (1994; the also cited paper by R. P. Kirshner has not appeared). The result depends strongly on how the bolometric luminosity is distributed over the spectrum. The so-called dilation factor is a major stumbling block. On different assumptions Baron et al. $(1995 ; 1996)$ have obtained $H_{0} \leq 50$. Obviously the method cannot be used at present for a quantitative discussion of $H_{0}$ (cf. also Nadyozhin 1998).

$4.9 H_{0}=73 \pm 6$ from the $D_{n}-\sigma$ method. Mould et al. (1997) have calibrated the $\mathrm{D}_{n}-\sigma$ data of Faber et al. (1989) using the Leo Group and the controversial Virgo and Fornax clusters as a zeropoint. Only the Leo group with three Cepheid distances is secure, but it provides with only two $\mathrm{D}_{n}-\sigma$ distances a shaky basis. If one adopts the Virgo cluster distance from Table 1 and $(m-M)=31.85$ as Fornax cluster modulus (4.3), an alternative calibration is obtained, leading to $H_{0}=63 \pm 6$. - It may be noted that the $\mathrm{D}_{n}-\sigma$ method applied to the bulges of $\mathrm{S} 0$ and $\mathrm{S}$ galaxies yields a high distance of the Virgo cluster (Table 1 ).

A recapitulation of Section 4.1-4.9 gives the following picture. 4.4 and 4.8 should be excluded as being too local and too controversial, respectively. 4.1, 4.2 and 4.5 depend entirely on the adopted small Virgo cluster 
distance, i.e. on the high weight given to the highly resolved galaxies on the near side of the cluster (4.1 depends also on the adopted high Virgo cluster velocity). 4.3 stands and falls with the assumption that the Cepheid distance of a single spiral (NGC1365) provides a useful mean distance of the E/S0 galaxies of the Fornax cluster. The same assumption affects 4.6 by about $15 \%$. The small Virgo and Fornax distances are essential for the high value of $H_{0}$ in 4.9. The remaining Section 4.7 is a textbook illustration of Malmquist bias.

\section{Conclusions}

A Test for the variation of $H_{0}$ with distance suggests a decrease by $\sim 7 \%$ from $1000<v \lesssim 18000 \mathrm{~km} \mathrm{~s}^{-1}$. At $v=10000 \mathrm{~km} \mathrm{~s}^{-1} H_{0}$ goes through a value close to the mean over very large scales.

A system of three interconnected distance scales (field galaxies, cluster distances relative to the Virgo cluster, and most significantly blue SNe Ia) give $H_{0}$ (cosmic) $=57 \pm 7$ (external error). Physical distance determinations from the SZ effect, gravitationally lensed quasars, and MWB fluctuations scatter about the same value.

A discussion of proposed high values of $H_{0}$ shows that disagreement focuses on two topics: 1) the true distance of the Virgo cluster, and 2) the appreciation of the Malmquist bias. One may add as item 3) the distance of the E/S0 galaxies in the Fornax cluster; the latter has lower priority because the peculiar motion of this cluster is unknown, and it is poorly tied into the relative distance scale of other clusters.

Acknowledgement: Financial support of the Swiss National Science Foundation is gratefully acknowledged. The author thanks his colleagues in the HST team for the luminosity calibration of SNe Ia, i.e. Dres. A. Sandage, A. Saha, L. Labhardt F.D. Macchetto, and N. Panagia, as well as the many collaborators behind the scenes at the STScI; much of the present understanding of $H_{0}$ depends on their work. He also thanks Mr. Bernd Reindl for his excellent help in all computational and technical matters.

\section{References}

Baron, E., Hauschildt, P. H., Branch, D., Austin, S., Garnavich, P., Ann, H. B., Wagner,

R. M., Filippenko, A. V., Matheson, T. and Liebert, J.: 1995, ApJ 441, 170.

Baron, E., Hauschildt, P. H., Nugent, P. and Branch, D.: 1996, MNRAS 283, 297.

Birkinshaw, M. and Hughes, J. P.: 1994, ApJ 420, 33.

Bottinelli, L., Gouguenheim, L., Paturel, G. H. and Teerikorpi, P.: 1986a, A\&A 156, 157.

Bottinelli, L., Gouguenheim, L., Paturel, G. H. and Teerikorpi, P.: 1986b, $A \& A$ 166, 39.

Bottinelli, L., Gouguenheim, L., Paturel, G. H. and Teerikorpi, P.: 1991, $A \& A$ 252, 550.

Branch, D.: 1998, ARAA, preprint.

Branch, D., Fisher, A. and Nugent, P.: 1993, AJ 106, 2383. 
Ciardullo, R., Jacoby, G. H., Feldmeier, J. J. and Bartlett, R. E.: 1998, ApJ 492, 62.

Di Benedetto, G. P.: 1997, ApJ 486, 60.

Faber, S., Wegner, G., Burstein, D., Davies, R., Dressler, A., Lynden-Bell, D. and Terlevich, R.: 1989, ApJS 69, 763.

Falco, E. E., Lehár, J. and Shapiro, I. I.: 1997, $A J$ 113, 540.

Feast, M.: 1995, Astrophys. Applications of Stellar Pulsations, ASP Conf. Series, 83, p. 209.

Federspiel, M.: 1998, in preparation.

Federspiel, M., Sandage, A. and Tammann, G. A.: 1994, ApJ 430, 29.

Federspiel, M., Tammann, G. A. and Sandage, A.: 1998, ApJ 495, 115.

Freedman, W. L., Madore, B. F. and Kennicutt, C.: 1997, in: The Extragalactic Distance Scale, eds. M. Livio, M. Donahue and N. Panagia, Cambridge: Cambridge University Press, p. 171.

Freedman, W. L., Mould, J. R., Kennicutt, R. C. and Madore, B. F.: 1998, this volume.

Giovanelli, R.: 1997a, in: The Extragalactic Distance Scale, eds. M. Livio, M. Donahue and N. Panagia, Cambridge: Cambridge University Press, p. 113.

Giovanelli, R.: 1997b, private communication.

Giovanelli, R., Haynes, M., da Costa, L. N., Freudling, W., Salzer, J. J. and Wegner, G.: 1997, ApJ 477, L1.

Goodwin, S. P., Gribbin, J. and Hendry, M. A.: 1997, AJ 114, 2212.

Gratton, R. G., Fusi Perci, F., Carretta, E., Clementini, G., Corsi, C. F. and Lattanzi, M.: 1997, ApJ 491, 749.

Hamuy, M., Phillips, M., Maza, J., Suntzeff, N., Schommer, R. and Aviles, R.: 1996, AJ 112, 2398.

Hendry, M. A. and Simmons, J. F. L.: 1990, $A \& A$ 237, 275.

Herbig, T., Lawrence, C. R. and Readhead, A. C. S.: 1995, ApJ 449, L5.

Höflich, P. and Khokhlov, A.: 1996, ApJ 457, 500.

Holzapfel, W. L. et al.: 1997, ApJ 480, 449.

Hubble, E.: 1929, Proc. Nat. Acad. Sci. 15, 168.

Hughes, S. M.: 1997, private communication.

Jerjen, H. and Tammann, G. A.: 1993, $A \& A$ 276, 1.

Keeton, C. R. and Kochanek, C. S.: 1997, $A p J$ 487, 42.

Kochanek, C. S.: 1997, ApJ 491, 13.

Kraan-Korteweg, R. C., Cameron, I. M. and Tammann, G. A.: 1988, ApJ 331, 620.

Kundić, T., Cohen, J. G., Blanford, R. D. and Lubin, L. M.: 1997, AJ 114, 507.

Laney, C. D. and Stobie, R. S.: 1992, in: Variable Stars and Galaxies, ed. B. Warner, ASP Conf. Series, 30, p. 119.

Lasenby, A. N. and Hancock, S.: 1995, in: Current Topics in Astrofundamental Physics: The Early Universe, eds. N. Sánchez and A. Zichichi, NATO Advanced Science Institutes Series, 467, Dordrecht: Kluwer Academic Publishers, p. 327.

Lasenby, A. N. and Jones, M. E.: 1997, in: The Extragalactic Distance Scale, eds. M. Livio, M. Donahue and N. Panagia, Cambridge: Cambridge University Press, p. 76.

Lauer, T. R. and Postman, M.: 1994, ApJ 425, 418.

Lee, M. G., Freedman, W. L. and Madore, B. F.: 1993, ApJ 417, 553.

Lineweaver, C. H.: 1998, this volume.

Lynden-Bell et al.: 1988, ApJ 326, 19.

Madore, B. and Freedman, W. L.: 1991, PASP 103, 933.

Madore, B. and Freedman, W. L.: 1998, ApJ 492, 110.

McHardy, L. M., Stewart, G. C., Edge, A. C., Cooke, B. A., Yamashita, K. and Hatsukade, I.: 1990, MNRAS 242, 215.

Mould, J., Sakai, S., Hughes, S. and Han, M.: 1997, in: The Extragalactic Distance Scale, eds. M. Livio, M. Donahue and N. Panagia, Cambridge: Cambridge University Press, p. 158.

Myers, S. T., Baker, J. E., Readhead, A. C. S., Leitch, E. M. and Herbig, T.: 1997, ApJ 485,1 . 
Nadyozhin, D. K.: 1998, preprint.

Nair, S.: 1996, in: Astrophysical Applications of Gravitational Lensing, eds. C. S. Kochanek and J. N. Hewitt, Dordrecht: Kluwer, p. 197.

Panagia, N. et al.: 1996, in: The Extragalactic Distance Scale, Poster Papers, eds. M. Livio, M. Donahue and N. Panagia, Baltimore: STScI, p. 54.

Parodi, B. and Tammann, G. A.: 1998, to be published.

Pont, F., Mayor, M., Turon, C. and VandenBerg, D. A.: 1998, A\&A 329, 87.

Reid, I. N.: 1998, $A J$ 115, 204.

Rephaeli, Y.: 1995, ARA $B A$ 33, 541.

Rephaeli, Y. and Yankovitch, D.: 1997, ApJ 481, L55.

Saha, A.: 1998, to be published.

Saha, A., Sandage, A., Labhardt, L., Tammann, G. A., Macchetto, F. D. and Panagia, P.: 1997, ApJ 486, 1.

Saio, H. and Gautschy, A.: 1998, $A p J$, in press.

Sandage, A.: 1988, PASP 100, 935.

Sandage, A.: 1993a, $A p J$ 106, 687 and 703.

Sandage, A.: 1993b, ApJ 402, 3.

Sandage, A.: 1993c, $A p J$ 404, 419.

Sandage, A.: 1994, ApJ 430, 13.

Sandage, A.: 1995, in: The Deep Universe, eds. B. Binggeli and R. Buser, Berlin: Springer, p. 1.

Sandage, A.: 1996a, $A J 111,1$.

Sandage, A.: 1996b, $A J$ 111, 18.

Sandage, A. and Cacciari, C.: 1990, ApJ 350, 645.

Sandage, A., Kristian, J. and Westphal, J. A.: 1976, ApJ 205, 688.

Sandage, A. and Tammann, G. A.: 1971, ApJ 167, 293.

Sandage, A. and Tammann, G. A.: 1997, in: Critical Dialogues on Cosmology Conference, ed. N. Turok, Princeton: Princeton University Press, p. 130.

Sandage, A. and Tammann, G. A.: 1998, MNRAS 293, L23.

Sandage, A., Tammann, G. A. and Federspiel, M.: 1995, ApJ 452, 1.

Schechter, P. L. et al.: 1997, ApJ 475, L85.

Schmidt, B. P. et al.: 1994, ApJ 432, 42.

Schröder, A.: 1996, PhD thesis, University of Basel.

Silbermann, N. et al.: 1998, $A p J$, in press.

Soffner, T. et al.: 1996, $A \& A$ 306, 9.

Tammann, G. A.: 1993, in: Planetary Nebulae, eds. R. Weinberger and A. Acker, IAU Symp., 155, Dordrecht: Kluwer, p. 515.

Tammann, G. A.: 1996, in: Reviews in Modern Astronomy 9, ed. R. E. Schielicke, Hamburg: Astronomische Gesellschaft, p. 139.

Tammann, G. A. and Federspiel, M.: 1997, in: The Extragalactic Distance Scale, eds. M. Livio, M. Donahue and N. Panagia, Cambridge: Cambridge University Press, p. 137.

Teerikorpi, P.: 1987, $A \& A 173,39$.

Theureau, G. et al.: 1997, $A \& A$ 322, 730.

Tonry, J. L.: 1997, in: The Extragalactic Distance Scale, eds. M. Livio, M. Donahue and N. Panagia, Cambridge: Cambridge University Press, p. 297.

Webster, M., Hobson, M. P., Lasenby, A. N., Lahav, O. and Rocha, G.: 1998, ApJL, submitted. 